\title{
Neurogranin and YKL-40: independent markers of synaptic degeneration and neuroinflammation in Alzheimer's disease
}

\author{
Konstantin Hellwig ${ }^{1}$, Hlin Kvartsberg ${ }^{2,3}$, Erik Portelius ${ }^{2}$, Ulf Andreasson ${ }^{2}$, Timo Jan Oberstein ${ }^{1}$, Piotr Lewczuk ${ }^{1,5}$,
} Kaj Blennow ${ }^{2}$, Johannes Kornhuber ${ }^{1}$, Juan Manuel Maler ${ }^{1}$, Henrik Zetterberg ${ }^{2,4}$ and Philipp Spitzer ${ }^{1 *}$ (D)

\begin{abstract}
Introduction: Neuroinflammation and synaptic degeneration are major neuropathological hallmarks in Alzheimer's disease (AD). Neurogranin and YKL-40 in cerebrospinal fluid (CSF) are newly discovered markers indicating synaptic damage and microglial activation, respectively.

Methods: CSF samples from 95 individuals including 39 patients with AD dementia (AD-D), 13 with mild cognitive impairment (MCl) due to $A D(M C l-A D), 29$ with $\mathrm{MCl}$ not due to $A D(M C l-o)$ and 14 patients with non-AD dementias (non-AD-D) were analyzed for neurogranin and YKL-40.

Results: Patients with dementia or MCI due to AD showed elevated levels of CSF neurogranin $(p<0.001$ for AD-D and $p<0.05$ for MCI-AD) and YKL-40 ( $p<0.05$ for AD-D and $p=0.15$ for MCl-AD) compared to mildly cognitively impaired subjects not diagnosed with AD. CSF levels of neurogranin and YKL-40 did not differ between MCl not due to $A D$ and non-AD dementias. In AD subjects no correlation between $Y K L-40$ and neurogranin was found. The CSF neurogranin levels correlated moderately with tau and p-tau but not with $A \beta_{42}$ or the MMSE in AD samples. No relevant associations between YKL-40 and MMSE or the core AD biomarkers, $A \beta_{42}$, t-tau and p-tau were found in $\mathrm{AD}$ subjects.
\end{abstract}

Conclusions: Neurogranin and YKL-40 are promising AD biomarkers, independent of and complementary to the established core AD biomarkers, reflecting additional pathological changes in the course of AD.

\section{Introduction}

Alzheimer's disease $(\mathrm{AD})$ is the most prevalent neurodegenerative disorder worldwide. The major pathological hallmarks of $\mathrm{AD}$ include extracellular depositions of $\beta$-amyloid $(A \beta)$ peptides as well as intracellular neurofibrillary tangles consisting of hyperphosphorylated tau, loss of synapses, and neuroinflammation [1,2]. The earliest pathophysiological events are expected to occur 10-20 years before the onset of dementia [3]. Changes in cerebrospinal fluid (CSF) biomarkers reflecting amyloid pathology $\left(\mathrm{A} \beta_{42}\right)$ and neurodegeneration [total tau $(\mathrm{t}-\mathrm{tau})$ and phosphorylated tau (p-tau)] occur early in the course of $\mathrm{AD}$ and are increasingly implicated in the early and

\footnotetext{
* Correspondence: philipp.spitzer@uk-erlangen.de

'Department of Psychiatry and Psychotherapy, University clinic Erlangen and Friedrich-Alexander University Erlangen-Nürnberg, Schwabachanlage 6,

91054 Erlangen, Germany

Full list of author information is available at the end of the article
}

predictive diagnosis of $\mathrm{AD}[4,5]$. The accuracy of diagnosis based on these core AD biomarkers is high, as long as markers of neurodegeneration and amyloidosis are altered concordantly [6]. However, in a proportion of patients, biomarker results may be contradictory, leading to lower diagnostic accuracy [7]. Additionally, $\mathrm{A} \beta_{42}$, t-tau, and $\mathrm{p}$ tau allow no conclusions about cognitive performance and only a limited prediction of cognitive decline to be made, a feature that is especially important for clinical trials [8]. Therefore, additional biomarkers reflecting further aspects of $\mathrm{AD}$ pathophysiology, such as synaptic degeneration and neuroinflammation, are needed. Loss of synapses is an early event in the course of $\mathrm{AD}$, and the correlation between synapse density and performance on neuropsychiatric tests such as the Mini Mental State Examination (MMSE) and verbal fluency tests is well established [9-12]. Neurogranin is a postsynaptic protein expressed in the neocortex, amygdala, caudate nucleus, putamen 
and hippocampus in the rodent brain [13]. In the human brain, expression is highest in associative cortical areas [14], suggesting a link with cognition. It is concentrated in dendritic spines of principal excitatory synapses, and its translocation to dendritic spines is impaired in AD [15-17]. Neurogranin levels are reduced in the hippocampus and cortex in $\mathrm{AD}$, indicating a loss of dendrites [2].

Synaptic proteins, including neurogranin, have been shown to be present in the CSF [18]. A first pilot study using immunoprecipitation (IP) and Western blot analysis showed a marked increase in CSF neurogranin levels in $\mathrm{AD}$ [19]. In a later study, using both IP-mass spectrometry and a newly developed immuno-based assay, researchers verified elevated levels of CSF neurogranin in a larger cohort of patients with $\operatorname{AD}[20,21]$. Importantly, high CSF neurogranin levels were also found in prodromal $\mathrm{AD}$ cases, and the degree of increase correlated with the rate of future cognitive decline [21].

Neuroinflammation is another common feature of AD pathology, and several epidemiological studies suggest a decrease in risk for $\mathrm{AD}$ after long-term administration of nonsteroidal anti-inflammatory drugs [22]. YKL-40, a $39 \mathrm{kDa}$ glycoprotein homologue to chitinase, is a marker for macrophage and microglial differentiation and activation [23-25]. Elevated CSF levels were shown in several infectious and noninfectious disorders of the central nervous system (CNS) [26]. Also, in AD, YKL-40 seems to be elevated in CSF [27-29]. The aim of this study was to investigate whether neurogranin as a marker for synaptic loss reflects cognitive disturbances and, together with YKL-40, shows aspects of AD pathophysiology complementary to amyloid pathology and neurodegeneration.

\section{Methods}

\section{Patients and sample collection}

The study protocol was approved by the ethics committee of the university clinic Erlangen-Nürnberg (number 3987), and all participants provided written informed consent. The patients were recruited in the memory clinic of the Department of Psychiatry and Psychotherapy in Erlangen, Germany. The participants underwent a physical, neurological, psychiatric, and neuropsychological examinations according to the Consortium to Establish a Registry for Alzheimer's Disease test battery [30]. Clinical diagnosis was supported by a brain magnetic resonance imaging scan, hexamethylpropyleneamine oxime single-photon emission computed tomography, and positive CSF biomarkers. Neurochemical dementia diagnosis was made using certified enzymelinked immunosorbent assay (ELISA) kits for $\mathrm{A} \beta_{1-40}$ (IBL International, Hamburg, Germany), $A \beta_{1-42}$ (The Genetics Company, Schlieren, Switzerland; and IBL International), and t-tau and p-tau (Fujirebio, Gent, Belgium; and IBL
International). Diagnoses of $\mathrm{AD}$ and mild cognitive impairment (MCI) were made according to the revised National Institute on Aging-Alzheimer's Association (NIA-AA) criteria $[4,5]$. None of the patients had a history indicative of hereditary AD. Subjects with malignant diseases or signs of systemic inflammation were excluded.

Experienced physicians collected the CSF samples by lumbar puncture in the L3-L4 or L4-L5 intervertebral space. With the exception that samples were centrifuged at $1500 \times g$ instead of $2000 \times g$, sampling and storage were carried out according to international consensus guidelines within 90 minutes after sampling [31].

\section{Neurogranin assay}

Measurement of neurogranin on the Meso Scale Discovery (MSD; Rockville, MD, USA) platform was performed as described previously [21]. The in-house monoclonal mouse antibody Ng7, which binds amino acids 52-65 of neurogranin, was used on a QUICKPLEX 96-well plate (MSD) as the capturing antibody. After blocking with $5 \%$ MSD Blocker for $1 \mathrm{~h}$ at room temperature (RT), the fulllength neurogranin calibrators in concentrations ranging from $31.3 \mathrm{pg} / \mathrm{ml}$ to $4000 \mathrm{pg} / \mathrm{ml}$, the blanks, and $50 \mu \mathrm{l}$ of CSF sample for each well were added in duplicates and coincubated overnight with a polyclonal anti-neurogranin antibody (ab 23570; EMD Millipore, Billerica, MA, USA). The next day, the plates were washed and SULFO-TAG goat anti-rabbit antibody (MSD) $(25 \mu \mathrm{l} /$ well $)$ was added for $2 \mathrm{~h}$ at RT. Before the plates were read on a QUICKPLEX SQ 120 reader (MSD), $150 \mu \mathrm{l}$ of $2 \times$ MSD read buffer with surfactant (MSD) was added to the wells. The samples were analyzed without knowledge of the clinical diagnosis. Intra-assay variation of the assay was calculated as the median of range/average from duplicate measurements, and the result was $10.4 \%$. Measurements of samples with a coefficient of variation $(\mathrm{CV})$ above $20 \%$ were repeated. The interassay $\mathrm{CV}$ was $14.2 \%$, as indicated by positive controls that were run on every plate. The detection ranges were $57.5-4000 \mathrm{pg} / \mathrm{ml}$ on the first plate and $69.4-4000 \mathrm{pg} / \mathrm{ml}$ on the second plate.

\section{YKL-40 assay}

YKL-40 levels were measured with a commercially available ELISA kit (R\&D Systems, Minneapolis, MN, USA) according to the manufacturer's instructions. This assay has been validated previously for CSF [28, 29, 32, 33]. For the YKL-40 analyses, the CSF was diluted 1:100. The samples were analyzed without knowledge of the clinical diagnosis. Intraassay CVs were all below $10 \%$. The interassay $\mathrm{CV}$, as indicated by positive controls run on every plate, was $5.6 \%$. 


\section{Statistical analysis}

The statistical analyses were performed with GraphPad Prism 6 software (GraphPad Software, La Jolla, CA, USA). Because data were skewed, group comparisons were made using the nonparametric Kruskal-Wallis test followed by Dunn's posttest. Correlations were determined using Spearman's rank correlation coefficient. Receiver operating characteristic (ROC) curves were drawn by plotting the false-positive fraction $(100 \%$ - specificity) versus the true-positive fraction (sensitivity). A $p$ value below 0.05 was considered significant.

\section{Results}

\section{Patient characteristics}

In total, 95 CSF samples collected at the Department of Psychiatry and Psychotherapy of the Universitätsklinikum Erlangen were included in the study and categorized according to the NIA-AA criteria. The cohort consisted of 39 patients with Alzheimer's disease dementia (AD-D) comprising patients with probable $\mathrm{AD}$ dementia with high evidence of $\mathrm{AD}$ pathophysiological process, patients with possible $\mathrm{AD}$ dementia with high evidence of $\mathrm{AD}$ pathophysiological process, and patients with probable $\mathrm{AD}$ dementia with intermediate evidence of AD pathophysiological process; 13 patients with $\mathrm{MCI}$ with a high likelihood that the mild cognitive impairment was due to Alzheimer's disease (MCI-AD); 29 patients with mild cognitive impairment unlikely due to Alzheimer's disease (MCI-o); and 14 patients with dementia unlikely due to AD (non-AD-D) (Table 1). The group of patients with other dementias included seven patients with frontotemporal dementia, three with vascular dementia, one with Lewy body dementia, and three with dementia of unknown origin. As AD biomarkers are applied mostly in the differential diagnosis of cognitive disturbances, MCI-o was chosen as the reference group. This group consisted especially of patients with depression, vascular disease, and early frontotemporal dementia (Table 1). The study population was well balanced overall according to age and sex. A significant difference in age was evident only between the non-AD-D and AD-D cohorts $(p<0.05)$. The core biomarkers $A \beta_{42}$, t-tau, and p-tau differed highly significantly in patients with MCI-AD and patients with AD compared with those in the MCI-o and non-AD-D groups $(p<0.001)$. As expected, MMSE scores in the non-AD-D and AD-D cohorts were significantly lower than in the $\mathrm{MCI}$ samples.

\section{Elevated levels of neurogranin in Alzheimer's disease}

Compared with patients with $\mathrm{MCI}-\mathrm{o}$, neurogranin was significantly increased in patients diagnosed with MCI$\mathrm{AD}(p<0.05)$ and those with $\mathrm{AD}$ dementia $(p<0.001)$. No difference was found between MCI-o and the nonAD-D patients (Fig. 1). The neurogranin levels in MCI-AD patients did not differ from those with suspected AD dementia. No difference in neurogranin levels was found within the different $\mathrm{AD}$ dementia populations separated by the certainty of the diagnosis (data not shown).

For the analysis of correlations, MCI-AD and AD-D were merged into an $\mathrm{AD}$ group and $\mathrm{MCI}-\mathrm{o}$ and non$\mathrm{AD}-\mathrm{D}$ were merged into a non-AD group. Neurogranin correlated with t-tau and p-tau in the non-AD group and in the AD group (Fig. 2). However, correlations were stronger within the non-AD group (Fig. 2). A correlation between neurogranin and $A \beta_{1-42}$ was found only in the non-AD group (Fig. 2). Interestingly, a strong correlation of neurogranin with $A \beta_{1-40}$ was also found (Fig. 2). The MMSE score did not correlate with neurogranin levels in any of the groups (Fig. 3).

Table 1 Patient characteristics

\begin{tabular}{|c|c|c|c|c|}
\hline & $\mathrm{MCl}-\mathrm{O}$ & Non-AD-D & $\mathrm{MCl}-\mathrm{AD}$ & AD-D \\
\hline Number of patients & 29 & 14 & 13 & 39 \\
\hline Age, yr & $69.4[61-75]$ & $65.1[59-71]$ & $73.3[69-77]$ & $72.5[68-76]^{a}$ \\
\hline Sex, M/F & $15 / 14$ & $6 / 8$ & $5 / 8$ & $18 / 21$ \\
\hline MMSE & 27 [26-28] & $20[20-23]^{b}$ & $26[25-28]^{c}$ & $21[19-24]^{b}$ \\
\hline$A \beta_{1-42}, \mathrm{pg} / \mathrm{ml}$ & 1262 [1014-1626] & 1255 [997-1585] & 638 [590-852] & 796 [618-928] \\
\hline$A \beta_{1-40}, \mathrm{pg} / \mathrm{ml}$ & $15,393[12,208-21,112]$ & 13,332 [9303-22,989] & $21612[17,875-26,191]$ & $20,803[15,168-24,448]$ \\
\hline t-tau, pg/ml & $226[158-246]$ & $242[189-320]$ & $580[487-789]$ & $522[403-708]$ \\
\hline p-tau, pg/ml & 44 [29-59] & $46[41-53]$ & 92 [80-113] & 99 [74-111] \\
\hline
\end{tabular}

A $\beta$-amyloid, $p$-tau phosphorylated tau, $t$-tau total tau, MMSE Mini Mental State Examination, $M C l$-o mild cognitive impairment not due to Alzheimer's disease, young control subjects without dementia, non- $A D-D$ dementia not due to Alzheimer's disease, $M C I-A D$ mild cognitive impairment due to $A \mid z h e i m e r$ 's disease, $A D-D$ Alzheimer's disease dementia

The values represent the median [interquartile range]

Differences between the groups were calculated using a nonparametric Kruskal-Wallis test followed by Dunn's posttest. No $p$ values were calculated for A $\beta$, $\mathrm{t}$-tau, and $\mathrm{p}$-tau, as the patients were selected according to these markers

${ }_{a} p<0.05$ vs. non-AD-D

${ }^{\mathrm{b}} p<0.001$ vs. $\mathrm{MCl}-\mathrm{o}$

${ }^{c} p<0.01$ vs. non-AD-D 


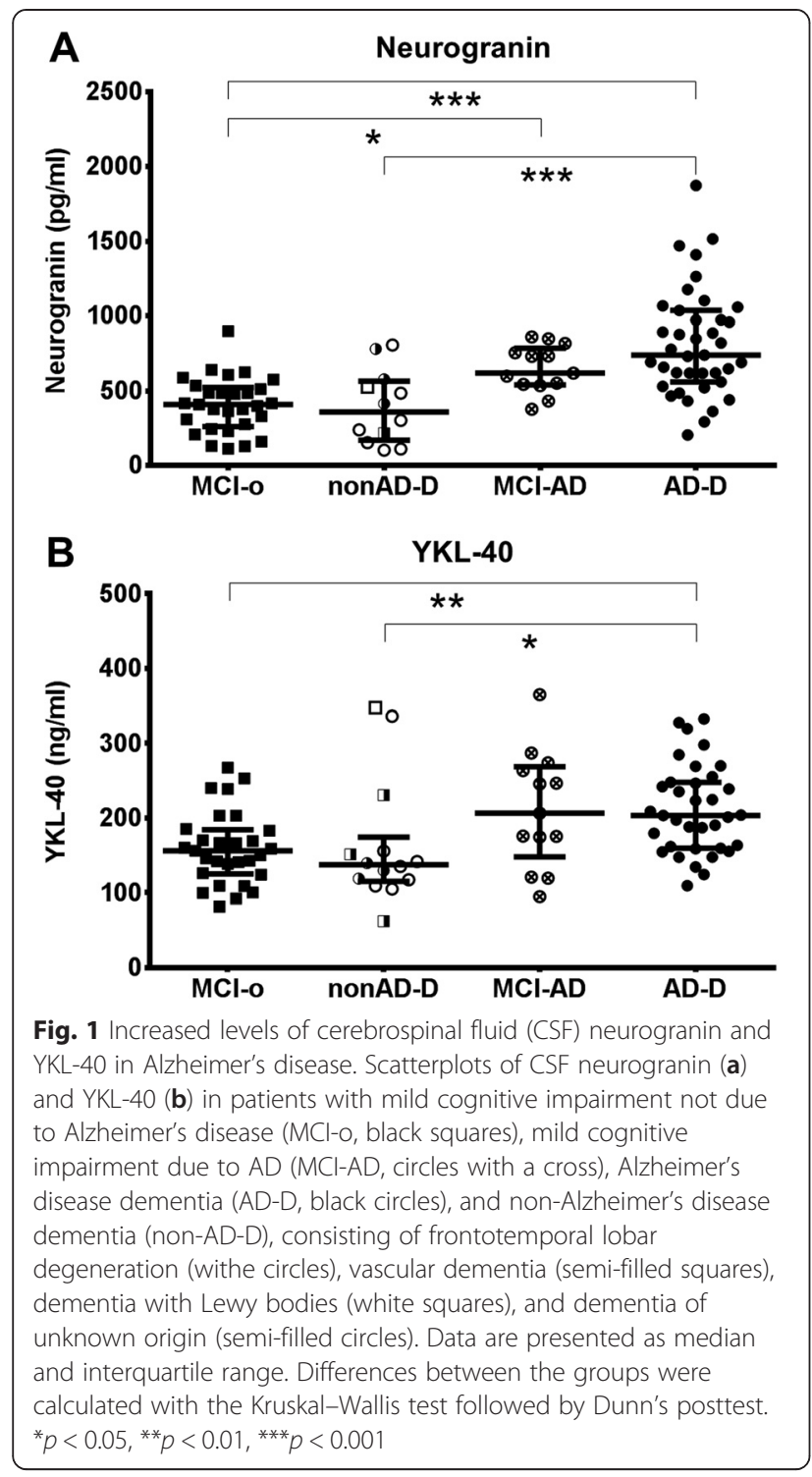

Elevated levels of YKL-40 in Alzheimer's disease

CSF YKL-40 levels were significantly elevated in patients with AD dementia as compared with those with MCI-o or non-AD dementia $(p<0.05)$. YKL-40 was also elevated in MCI-AD patients, but without reaching statistical significance $(p=0.15)$. The YKL-40 levels of MCI-AD and AD dementia patients did not differ (Fig. 1). The patients with other forms of dementia did not show an elevation in YKL-40 levels compared with MCI-o patients (Fig. 1).

YKL-40 was age-correlated in our sample (Additional file 1: Figure S1). However, as the populations with cognitive disturbances were age-matched, no statistical correction for age was made. A significant correlation of YKL-40 with t-tau and p-tau was found only in the nonAD group (Additional file 2: Figure $\mathrm{S} 2$ ). In addition, no correlation of YKL-40 with $A \beta_{1-42}, A \beta_{1-40}$, or MMSE score was observed (Fig. 3 and Additional file 2: Figure S2).

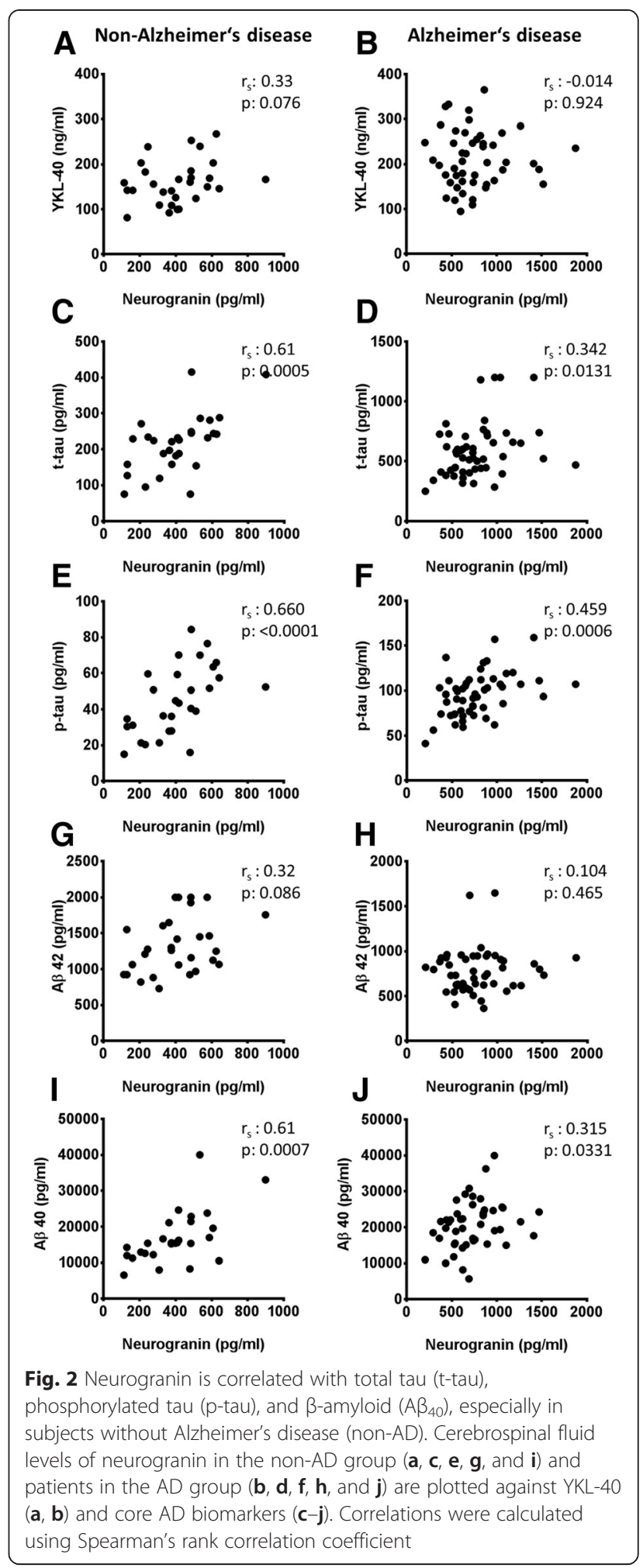

No correlation between neurogranin and YKL-40

No significant correlation could be shown between neurogranin and YKL-40, as indicators for postsynaptic integrity and microglial activation in AD (Fig. 2). 

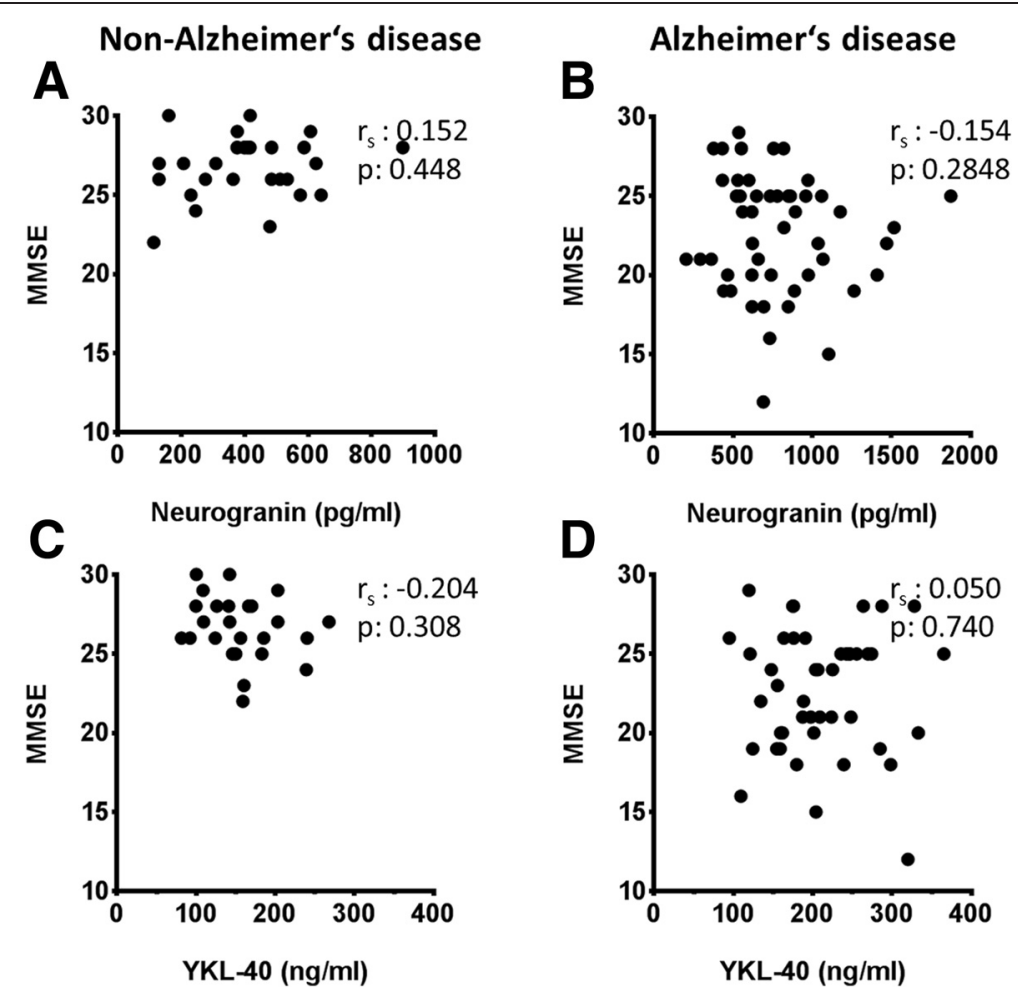

Fig. 3 Neurogranin and YKL-40 are not correlated with Mini Mental State Examination (MMSE) scores. Cerebrospinal fluid levels of neurogranin $(\mathbf{a}, \mathbf{b})$ and YKL-40 (c, d) are plotted against MMSE scores in the non-AD group $(\mathbf{a}, \mathbf{c})$ and the AD group (b, d). Correlations were calculated using Spearman's rank correlation coefficient

Neurogranin supports the early and differential diagnosis of $A D$

To test the suitability of neurogranin and YKL-40 as biomarkers for AD, ROC curves were calculated. Samples of patients with $\mathrm{AD}$ pathology (AD group) could be separated from those without signs of AD pathology, including other dementias (non-AD group), with an area under the curve (AUC) of 0.85 for neurogranin and 0.66 for YKL-40 (Fig. 4). Combining the two markers by multiplication resulted in an AUC of 0.85 (Fig. 4).

\section{Discussion}

We have shown that the synaptic protein neurogranin and YKL-40 are elevated in the CSF of patients with AD. Even though both markers were significantly increased, they did not correlate with each other in AD.

In the diagnosis of cognitive disturbances, biochemical markers as indicators of the disease are increasingly implicated. Unfortunately, biochemical markers reflecting cognitive decline are still sparse [8]. It has long been known that the number of synapses is well correlated with the degree of cognitive disturbances [10, 34, 35]. Therefore, it is expected that biomarkers indicating synaptic integrity would be well suited to reflect cognitive decline. In our study, CSF neurogranin levels were elevated in $\mathrm{AD}$. However, we found no difference in the

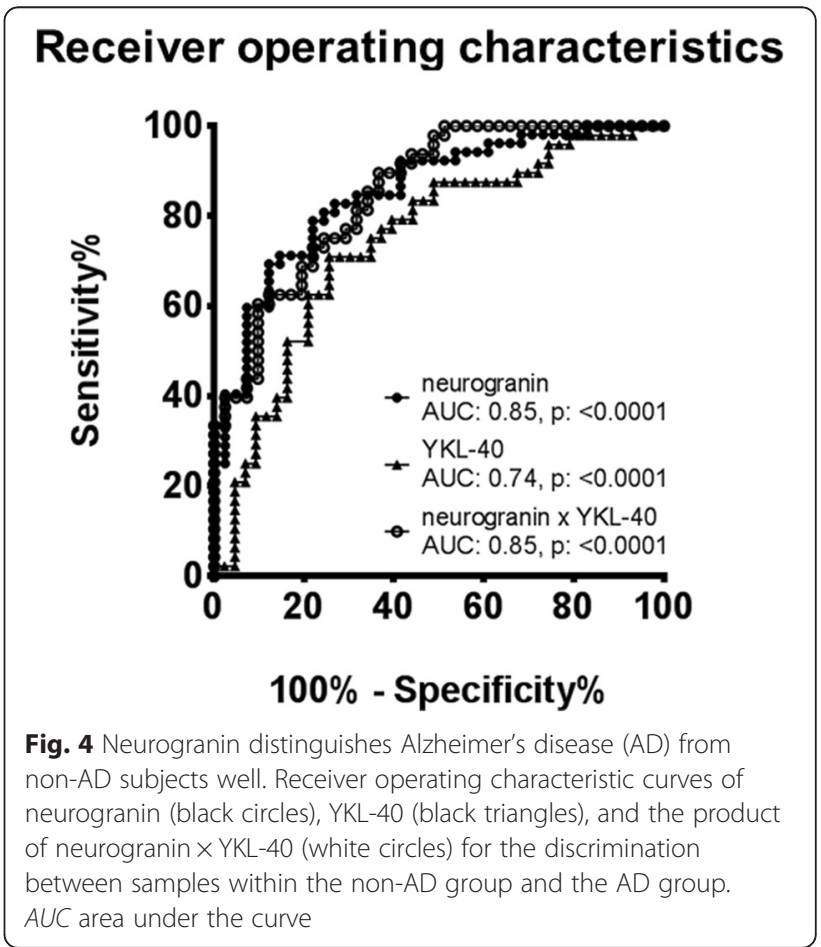


levels of neurogranin in the dementia stage versus MCI. In addition, there was no correlation between neurogranin levels and MMSE scores. Thus, our results are in line with previous reports of elevated levels of neurogranin in $\mathrm{AD}[19,20,36,37]$. In contrast to our present study, Thorsell et al. did not distinguish between MCI due to $\mathrm{AD}$ and $\mathrm{MCI}$ due to other diseases, and they measured neurogranin levels in the MCI group between that of controls and that of patients with AD [19]. In their study, Kvartsberg et al. included a neuropsychological follow-up investigation which showed that high CSF levels of neurogranin at baseline predicted a more rapid decline in cognition [20]. This might indicate that neurogranin reflects not the synaptic density but rather the intensity of current synaptic destruction.

In line with previous studies, we have shown that neurogranin differentiated well between $\mathrm{AD}$ and other neurodegenerative diseases. Established core biomarkers (i.e., $\mathrm{A} \beta_{1-42}, \mathrm{t}$-tau, and $\mathrm{p}$-tau) have high diagnostic accuracy in discriminating individuals with AD from subjects without cognitive disturbances, but their diagnostic performance in differentiating $\mathrm{AD}$ from other dementias is far from optimal [38]. Interestingly, CSF neurogranin was not elevated in our cohort of patients with other neurodegenerative diseases. However, the cohort of nonAD-D patients was small and comprised especially patients with frontotemporal lobar degeneration. Further research is necessary to clarify whether the elevation of neurogranin is specific for AD.

The stronger correlation of neurogranin and tau/p-tau in non-AD patients as compared with patients with $\mathrm{AD}$ and the missing elevation of neurogranin in non-AD-D patients also points to a mechanism of neurodegeneration in $\mathrm{AD}$ distinct from the physiological dying of neurons and distinct from other neurodegenerative diseases. Most likely, it shows a degeneration of synapses that is weakly related to the axonal damage indicated by tau [39]. The exact mechanism by which neurogranin is released is unclear.

Elevated levels of CSF YKL-40 in early stages of AD have been demonstrated in two independent studies, but there are also contradictory data [28, 32, 40]. In our study, we confirmed that YKL-40 is elevated early in the course of $\mathrm{AD}$ and that the levels do not change during disease progression. In addition, YKL-40 levels in other dementias did not differ from those with MCI not due to $\mathrm{AD}$. This suggests that neuroinflammation in $\mathrm{AD}$ pathology differs from that in other dementias. In accordance with the concept of inflammaging, introduced by Franceschi et al., we found a correlation of YKL- 40 with age. Inflammaging describes a low-grade, chronic upregulation of inflammatory responses during aging as a risk factor for several age-dependent diseases [41, 42]. Accumulating evidence shows a similar alteration in the
CNS of the elderly as a prodrome of $\mathrm{AD}$ [43]. In part, this increased immune reactivity in the aged brain might be derived from primed microglia. Primed microglia are in a preactivated state and tend to react in a prolonged manner and by secretion of higher amounts of proinflammatory signals [44]. Excessive inflammatory responses by primed microglia aggravate neurodegeneration, impair synaptic plasticity, and lead to cognitive decline [45]. However, we did not find a correlation between YKL-40 and MMSE. Yet, as a marker for microglial activation, YKL-40 seems well suited to reflect these aspects of $A D$ pathophysiology.

Even though a link between microglial activation and synaptic degeneration can be postulated, we found no correlation between neurogranin and YKL-40 in our study. As detailed above, YKL-40 is a rather unspecific marker that is highly influenced by patients' comorbidities. This might also explain why data on YKL-40 correlations are somewhat contradictory. Two studies showed a correlation with $\mathrm{p}$-tau and $\mathrm{t}$-tau, whereas a third did not find any correlation with CSF tau levels [27, 29, 40]. Data on correlations with MMSE are likewise conflicting $[29,40]$. The missing correlation between neurogranin and YKL-40 suggests that these two markers reflect two different aspects of neurodegeneration in AD. Whereas YKL-40 might represent A $\beta$-mediated activation of microglia and neuroinflammation, elevated levels of neurogranin might indicate synaptic damage of another origin, such as direct $A \beta$-mediated neurotoxicity via soluble oligomers, disturbances in calcium homeostasis, or mitochondrial damage [46-52].

To evaluate neurogranin and YKL-40 as potential biomarkers for $\mathrm{AD}$, we determined ROC curves for both markers alone and a combination of both markers by multiplication. With an AUC of 0.85, the diagnostic performance of neurogranin is in the reported range of the isolated core biomarkers. The combination of $A \beta$, tau, and neurogranin might therefore improve diagnostic performance considerably. A comparison with core biomarkers was not possible in our study, as patients were selected according to these markers. To further evaluate the potential of neurogranin as a diagnostic biomarker, further studies including patients not stratified by established biomarkers are needed. The additional benefit of YKL-40 as biomarker for AD is limited, with an AUC of 0.66 , and is a rather unspecific marker. However, YKL40 might be useful for patient stratification and monitoring of drugs targeting microglial activation.

\section{Conclusions}

Taken together, elevated levels of neurogranin and YKL40 could be found in CSF samples of patients with AD compared with those with other dementias and control subjects. The expected relationship between postsynaptic 
damage and microglial activation in $\mathrm{AD}$ could not be shown using these markers. Therefore, neurogranin and YKL-40 might support the biochemical dementia diagnosis by reflecting aspects of AD pathophysiology complementary to $A \beta$ and tau.

\section{Additional files}

Additional file 1: Figure S1. YKL-40 is strongly, and neurogranin weakly, correlated with age. CSF levels of neurogranin and YKL-40 are plotted against age in the whole sample. Correlations were calculated with Spearman's rank correlation coefficient. (TIF $106 \mathrm{~kb}$ )

Additional file 2: Figure S2. YKL-40 is correlated with t-tau and p-tau in non-AD subjects. CSF levels of YKL-40 in the non-AD group (A, C, E, G) and the $A D$ group $(B, D, F, H)$ are plotted against core $A D$ biomarkers. Correlations were calculated with Spearman's rank correlation coefficient. (TIF $343 \mathrm{~kb})$

\section{Abbreviations}

AB: $\beta$-amyloid; AD: Alzheimer's disease; AD-D: Alzheimer's disease dementia; AD group: patients with Alzheimer's disease (including mild cognitive impairment and dementia); AUC: Area under the curve; CNS: central nervous system; CSF: cerebrospinal fluid; CV: coefficient of variation; ELISA: enzymelinked immunosorbent assay; IP: immunoprecipitation; MCl: mild cognitive impairment; MCI-AD: mild cognitive impairment due to Alzheimer's disease; MCl-o: mild cognitive impairment not due to Alzheimer's disease (MCl-other); MMSE: Mini Mental State Examination; MSD: Meso Scale Discovery; NIA-AA: National Institute on Aging-Alzheimer's Association; non-AD-D: dementia not due to Alzheimer's disease; non-AD group: patients without Alzheimer's disease (including mild cognitive impairment and dementia); p-tau: phosphorylated tau; ROC: receiver operating characteristic; RT: room temperature; $\mathrm{t}$-tau: total tau.

\section{Competing interests}

KB has served on advisory boards for Eli Lilly and Company, IBL International, and Roche Diagnostics and has given lectures for Fujirebio Europe. KB and $\mathrm{HZ}$ are cofounders of Brain Biomarker Solutions AB (Gothenburg, Sweden), a GU Ventures-based platform company at the University of Gothenburg. $\mathrm{PL}$ has received consultation and lecture honoraria from Innogenetics, IBL International, AJ Roboscreen, Beckman Coulter, and Roche and holds the position of visiting professor at the Medical University of Białystok (Białystok, Poland). KH, HK, EP, UA, TJO, JK, JMM, and PS declare that they have no competing interests.

\section{Authors' contributions}

$\mathrm{KH}, \mathrm{PL}, \mathrm{KB}, J \mathrm{MM}, \mathrm{HZ}$, and PS designed the study. PL, JMM, TJO, JK, and PS investigated the patients and collected the samples. $\mathrm{KH}$ and $\mathrm{HK}$ carried out the experiments. EP, UA, KB, PL, and $\mathrm{HZ}$ supervised and substantially supported the acquisition of data based on their vast experience. KH, EP, $\mathrm{KB}, \mathrm{UA}, \mathrm{HZ}$, and PS carried out statistical analysis. $\mathrm{KH}$ and PS drafted the manuscript. All authors reviewed the manuscript critically and provided constructive comments to improve the quality of the manuscript. All authors read and approved the final manuscript.

\section{Acknowledgments}

This work was supported by grants from the Interdisciplinary Center for Clinical Research (IZKF; Erlangen, Germany), the Swedish Brain Foundation (Hjärnfonden), and the Torsten Söderberg Foundation at the Royal Swedish Academy of Sciences. PL is supported by the German Bundesministerium für Bildung und Forschung (grant 01ED1203D) within the Biomarkers for Alzheimer's disease and Parkinson's disease (BIOMARKAPD) project of the EU Joint Programme - Neurodegenerative Disease Research (JPND). The research leading to these results was supported by the Innovative Medicines Initiative joint undertaking under grant agreement number 115372, the resources of which are composed of financial contributions from the European Union's Seventh Framework Programme (FP7/2007-2013) and European Federation of Pharmaceutical Industries and Associations member companies' in-kind contributions. The present work was performed in fulfillment of KH's requirements for obtaining the "Dr. med." Degree. We acknowledge support from Deutsche Forschungsgemeinschaft (DFG) and Friedrich-Alexander-Universität Erlangen-Nürnberg (FAU) within the funding program Open Access Publishing.

\section{Author details}

${ }^{1}$ Department of Psychiatry and Psychotherapy, University clinic Erlangen and Friedrich-Alexander University Erlangen-Nürnberg, Schwabachanlage 6, 91054 Erlangen, Germany. ${ }^{2}$ Clinical Neurochemistry Laboratory, Institute of Neuroscience and Physiology, Department of Psychiatry and Neurochemistry, Sahlgrenska Academy at the University of Gothenburg, Mölndal, Sweden.

${ }^{3}$ AlzeCure Foundation, Karolinska Institutet Science Park, Huddinge, Sweden. ${ }^{4}$ Department of Molecular Neuroscience, UCL Institute of Neurology, London WC1N 3BG, UK. ${ }^{5}$ Department of Neurodegeneration Diagnostics, Medical University of Białystok, Białystok, Poland.

Received: 14 July 2015 Accepted: 28 October 2015

Published online: 24 December 2015

\section{References}

1. Perl DP. Neuropathology of Alzheimer's disease. Mt Sinai J Med. 2010;77:32-42. doi:10.1002/msj.20157.

2. Davidsson P, Blennow K. Neurochemical dissection of synaptic pathology in Alzheimer's disease. Int Psychogeriatr. 1998;10:11-23.

3. Jack CR Jr, Knopman DS, Jagust WJ, Shaw LM, Aisen PS, Weiner MW, et al. Hypothetical model of dynamic biomarkers of the Alzheimer's pathological cascade. Lancet Neurol. 2010;9:119-28.

4. McKhann GM, Knopman DS, Chertkow H, Hyman BT, Jack CR Jr, Kawas CH, et al. The diagnosis of dementia due to Alzheimer's disease: recommendations from the National Institute on Aging-Alzheimer's Association workgroups on diagnostic guidelines for Alzheimer's disease. Alzheimers Dement. 2011;7:263-9. doi:10.1016/j.jalz.2011.03.005.

5. Albert MS, DeKosky ST, Dickson D, Dubois B, Feldman HH, Fox NC, et al. The diagnosis of mild cognitive impairment due to Alzheimer's disease: recommendations from the National Institute on Aging-Alzheimer's Association workgroups on diagnostic guidelines for Alzheimer's disease. Alzheimers Dement. 2011;7:270-9.

6. Hansson O, Zetterberg H, Buchhave P, Londos E, Blennow K, Minthon L. Association between CSF biomarkers and incipient Alzheimer's disease in patients with mild cognitive impairment: a follow-up study. Lancet Neurol. 2006;5:228-34. doi:10.1016/S1474-4422(06)70355-6.

7. Oksengard AR, Cavallin L, Axelsson R, Andersson C, Nagga K, Winblad B, et al. Lack of accuracy for the proposed 'Dubois criteria' in Alzheimer's disease: a validation study from the Swedish brain power initiative. Dement Geriatr Cogn Disord. 2010;30:374-80. doi:10.1159/000321121.

8. Drago V, Babiloni C, Bartres-Faz D, Caroli A, Bosch B, Hensch T, et al. Disease tracking markers for Alzheimer's disease at the prodromal (MCl) stage. J Alzheimers Dis. 2011;26:159-99.

9. Masliah E, Mallory M, Alford M, DeTeresa R, Hansen LA, McKeel DW Jr, et al. Altered expression of synaptic proteins occurs early during progression of Alzheimer's disease. Neurology. 2001;56:127-9.

10. Scheff SW, Price DA, Schmitt FA, DeKosky ST, Mufson EJ. Synaptic alterations in CA1 in mild Alzheimer disease and mild cognitive impairment. Neurology. 2007;68:1501-8. doi:10.1212/01.wnl.0000260698.46517.8f.

11. Scheff SW, Price DA, Schmitt FA, Scheff MA, Mufson EJ. Synaptic loss in the inferior temporal gyrus in mild cognitive impairment and Alzheimer's disease. J Alzheimers Dis. 2011;24:547-57. doi:10.3233/JAD-2011-101782.

12. Scheff SW, Price DA, Ansari MA, Roberts KN, Schmitt FA, Ikonomovic MD, et al. Synaptic change in the posterior cingulate gyrus in the progression of Alzheimer's disease. J Alzheimers Dis. 2015;43:1073-90. doi:10.3233/JAD-141518.

13. Represa A, Deloulme JC, Sensenbrenner M, Ben-Ari Y, Baudier J, Neurogranin: immunocytochemical localization of a brain-specific protein kinase C substrate. J Neurosci. 1990;10:3782-92.

14. Bogdanovic N, Davidsson P, Gottfries J, Volkman I, Winblad B, Blennow K. Regional and cellular distribution of synaptic proteins in the normal human brain. Brain Aging Int J. 2002;5:18-30.

15. Singec $I$, Knoth R, Ditter M, Volk B, Frotscher M. Neurogranin is expressed by principal cells but not interneurons in the rodent and monkey neocortex and hippocampus. J Comp Neurol. 2004;479:30-42. doi:10.1002/cne.20302.

16. Guadano-Ferraz A, Vinuela A, Oeding G, Bernal J, Rausell E. RC3/neurogranin is expressed in pyramidal neurons of motor and somatosensory cortex in 
normal and denervated monkeys. J Comp Neurol. 2005;493:554-70. doi:10.1002/cne.20774.

17. Chang JW, Schumacher E, Coulter PM 2nd, Vinters HV, Watson JB. Dendritic translocation of RC3/neurogranin mRNA in normal aging, Alzheimer disease and fronto-temporal dementia. J Neuropathol Exp Neurol. 1997;56:1 105-18.

18. Davidsson P, Puchades M, Blennow K. Identification of synaptic vesicle, pre- and postsynaptic proteins in human cerebrospinal fluid using liquid-phase isoelectric focusing. Electrophoresis. 1999;20:431-7. doi:10.1002/(SICI)1522-2683(19990301)20:3<431::AID-ELPS431>3.0.CO;2-2.

19. Thorsell A, Bjerke M, Gobom J, Brunhage E, Vanmechelen E, Andreasen N, et al. Neurogranin in cerebrospinal fluid as a marker of synaptic degeneration in Alzheimer's disease. Brain Res. 2010;1362:13-22. doi:10.1016/j.brainres.2010.09.073.

20. Kvartsberg H, Duits FH, Ingelsson M, Andreasen N, Öhrfelt A, Andersson K, et al. Cerebrospinal fluid levels of the synaptic protein neurogranin correlates with cognitive decline in prodromal Alzheimer's disease. Alzheimers Dement. 2015;11:1180-90. doi:10.1016/j.jalz.2014.10.009.

21. Kvartsberg H, Portelius $E$, Andreasson U, Brinkmalm G, Hellwig K, Lelental N, et al. Characterization of the postsynaptic protein neurogranin in paired cerebrospinal fluid and plasma samples from Alzheimer's disease patients and healthy controls. Alzheimers Res Ther. 2015;7:40.

22. McGeer PL, McGeer EG. The amyloid cascade-inflammatory hypothesis of Alzheimer disease: implications for therapy. Acta Neuropathol. 2013;126:479-97. doi:10.1007/s00401-013-1177-7.

23. Hakala BE, White C, Recklies AD. Human cartilage gp-39, a major secretory product of articular chondrocytes and synovial cells, is a mammalian member of a chitinase protein family. J Biol Chem. 1993;268:25803-10.

24. Rehli M, Niller HH, Ammon C, Langmann S, Schwarzfischer $L$, Andreesen $R$ et al. Transcriptional regulation of $C H I 3 L 1$, a marker gene for late stages of macrophage differentiation. J Biol Chem. 2003;278:44058-67. doi:10.1074/jbc.M306792200.

25. Bonneh-Barkay D, Bissel SJ, Wang G, Fish KN, Nicholl GC, Darko SW, et al. YKL-40, a marker of simian immunodeficiency virus encephalitis, modulates the biological activity of basic fibroblast growth factor. Am J Pathol. 2008;173:130-43. doi:10.2353/ajpath.2008.080045.

26. Prakash M, Bodas M, Prakash D, Nawani N, Khetmalas M, Mandal A, et al. Diverse pathological implications of YKL-40: answers may lie in 'outside-in' signaling. Cell Signal. 2013;25:1567-73. doi:10.1016/j.cellsig.2013.03.016.

27. Craig-Schapiro R, Perrin RJ, Roe CM, Xiong C, Carter D, Cairns NJ, et al. YKL-40: a novel prognostic fluid biomarker for preclinical Alzheimer's disease. Biol Psychiatry. 2010;68:903-12. doi:10.1016/j.biopsych.2010.08.025.

28. Olsson B, Hertze J, Lautner R, Zetterberg H, Nagga K, Hoglund K, et al. Microglial markers are elevated in the prodromal phase of Alzheimer's disease and vascular dementia. J Alzheimers Dis. 2013;33:45-53. doi:10.3233/JAD-2012-120787

29. Rosen C, Andersson CH, Andreasson U, Molinuevo JL, Bjerke M, Rami L, et al. Increased levels of chitotriosidase and YKL-40 in cerebrospinal fluid from patients with Alzheimer's disease. Dement Geriatr Cogn Dis Extra. 2014:4:297-304. doi:10.1159/000362164.

30. Morris JC, Mohs RC, Rogers H, Fillenbaum G, Heyman A. Consortium to establish a registry for Alzheimer's disease (CERAD) clinical and neuropsychological assessment of Alzheimer's disease. Psychopharmacol Bull. 1988;24:641-52.

31. Teunissen CE, Tumani H, Engelborghs S, Mollenhauer B. Biobanking of CSF: international standardization to optimize biomarker development. Clin Biochem. 2014;47:288-92.

32. Mattsson N, Tabatabaei S, Johansson P, Hansson O, Andreasson U, Mansson JE, et al. Cerebrospinal fluid microglial markers in Alzheimer's disease: elevated chitotriosidase activity but lack of diagnostic utility. Neuromolecular Med. 2011;13:151-9.

33. Malmestrom C, Axelsson M, Lycke J, Zetterberg H, Blennow K, Olsson B. CSF levels of YKL-40 are increased in MS and replaces with immunosuppressive treatment. J Neuroimmunol. 2014;269:87-9.

34. Blennow K, Bogdanovic N, Alafuzoff I, Ekman R, Davidsson P. Synaptic pathology in Alzheimer's disease: relation to severity of dementia, but not to senile plaques, neurofibrillary tangles, or the ApoE4 allele. J Neural Transm. 1996;103:603-18.

35. Terry RD, Masliah E, Salmon DP, Butters N, DeTeresa R, Hill R, et al. Physical basis of cognitive alterations in Alzheimer's disease: synapse loss is the major correlate of cognitive impairment. Ann Neurol. 1991;30:572-80. doi:10.1002/ana.410300410.
36. De Vos A, Jacobs D, Struyfs H, Fransen E, Andersson K, Portelius E, et al. C-terminal neurogranin is increased in cerebrospinal fluid but unchanged in plasma in Alzheimer's disease. Alzheimers Dement. 2015. doi:10.1016/j.jalz.2015.05.012.

37. Kester MI, Teunissen CE, Crimmins DL, Herries EM, Ladenson JH, Scheltens P, et al. Neurogranin as a cerebrospinal fluid biomarker for synaptic loss in symptomatic Alzheimer disease. JAMA Neurol. 2015;72:1275-80.

38. Blennow K, Hampel H, Weiner M, Zetterberg H. Cerebrospinal fluid and plasma biomarkers in Alzheimer disease. Nat Rev Neurol. 2010;6:131-44. doi:10.1038/nrneurol.2010.4.

39. Abdelhak A, Junker A, Brettschneider J, Kassubek J, Ludolph AC, Otto M, et al. Brain-specific cytoskeletal damage markers in cerebrospinal fluid: is there a common pattern between amyotrophic lateral sclerosis and primary progressive multiple sclerosis? Int J Mol Sci. 2015;16:17565-88. doi:10.3390/ijms160817565.

40. Antonell A, Mansilla A, Rami L, Llado A, Iranzo A, Olives J, et al. Cerebrospinal fluid level of YKL-40 protein in preclinical and prodromal Alzheimer's disease. J Alzheimers Dis. 2014;42:901-8. doi:10.3233/JAD-140624.

41. Franceschi C, Capri M, Monti D, Giunta S, Olivieri F, Sevini F, et al. Inflammaging and anti-inflammaging: a systemic perspective on aging and longevity emerged from studies in humans. Mech Ageing Dev. 2007;128:92-105. doi:10.1016/j.mad.2006.11.016.

42. Franceschi C, Campisi J. Chronic inflammation (inflammaging) and its potential contribution to age-associated diseases. J Gerontol A Biol Sci Med Sci. 2014;69:S4-9. doi:10.1093/gerona/glu057.

43. Giunta B, Fernandez F, Nikolic WV, Obregon D, Rrapo E, Town T, et al. Inflammaging as a prodrome to Alzheimer's disease. J Neuroinflammation. 2008;5:51. doi:10.1186/1742-2094-5-51.

44. Perry VH, Teeling J. Microglia and macrophages of the central nervous system: the contribution of microglia priming and systemic inflammation to chronic neurodegeneration. Semin Immunopathol. 2013;35:601-12. doi:10.1007/s00281-013-0382-8.

45. Norden DM, Muccigrosso MM, Godbout JP. Microglial priming and enhanced reactivity to secondary insult in aging, and traumatic CNS injury, and neurodegenerative disease. Neuropharmacology. 2015;96:29-41. doi:10.1016/j.neuropharm.2014.10.028.

46. Perry VH, Nicoll JA, Holmes C. Microglia in neurodegenerative disease. Nat Rev Neurol. 2010;6:193-201. doi:10.1038/nrneurol.2010.17.

47. Block ML. NADPH oxidase as a therapeutic target in Alzheimer's disease. BMC Neurosci. 2008;9:8. doi:10.1186/1471-2202-9-S2-S8.

48. Wilcox KC, Lacor PN, Pitt J, Klein WL. A oligomer-induced synapse degeneration in Alzheimer's disease. Cell Mol Neurobiol. 2011;31:939-48. doi:10.1007/s10571-011-9691-4.

49. Mattson MP. Calcium and neurodegeneration. Aging Cell. 2007;6:337-50. doi:10.1111/j.1474-9726.2007.00275.x.

50. Kuchibhotla KV, Goldman ST, Lattarulo CR, Wu HY, Hyman BT, Bacskai BJ. $A \beta$ plaques lead to aberrant regulation of calcium homeostasis in vivo resulting in structural and functional disruption of neuronal networks. Neuron. 2008:59:214-25. doi:10.1016/j.neuron.2008.06.008.

51. Reddy PH. Amyloid beta, mitochondrial structural and functional dynamics in Alzheimer's disease. Exp Neurol. 2009;218:286-92. doi:10.1016/j.expneurol.2009.03.042.

52. Haass C, Selkoe DJ. Soluble protein oligomers in neurodegeneration: lessons from the Alzheimer's amyloid $\beta$-peptide. Nat Rev Mol Cell Biol. 2007;8:101-12. doi:10.1038/nrm2101. 